\title{
ON SUPPORTS OF SEMIGROUPS OF MEASURES
}

\author{
by H. L. CHOW \\ (Received 17th January 1973)
}

Let $S$ denote a compact semitopological semigroup (i.e. the multiplication is separately continuous) and $P(S)$ the set of probability measures on $S$. Then $P(S)$ is a compact semitopological semigroup under convolution and the weak * topology (4). Let $\Gamma$ be a subsemigroup of $P(S)$ and $S(\Gamma)=\bigcup_{\mu \in \Gamma}$ supp $\mu$ where supp $\mu$ is the support of $\mu \in P(S)$. In the case in which $S$ is commutative it was shown by Glicksberg in (4) that $S(\Gamma)$ is an algebraic group in $S$ if $\Gamma$ is an algebraic group. For a general semigroup $S$, Pym (7) considered $\Gamma=\{\eta\}$, $\eta$ being an idempotent, and established that $S(\Gamma)$ is a topologically simple subsemigroup of $S$, i.e. every ideal of $S(\Gamma)$ is dense in $S(\Gamma)$. In this note we prove that if $\Gamma$ is a simple subsemigroup of $P(S)$ (a semigroup is simple if it contains no proper ideal) which contains an idempotent then $S(\Gamma)$ is a topologically simple subsemigroup of $S$. We also give an example to show that our conclusion (hence also Pym's) is best possible in the sense that $S(\Gamma)$ is not simple in general.

Next, if $S$ is jointly continuous and supp $\Gamma=\bar{S}(\Gamma)$ (the bar denotes closure), we can then obtain that supp $\Gamma$ must be simple if $\Gamma$ is assumed simple but not necessarily containing an idempotent. In other words, (Theorem 4) for a jointly continuous semigroup $S$, the support of a simple subsemigroup of $P(S)$ is a compact simple subsemigroup of $S$. This fills a lacuna in the literature. In fact it was first stated by Lin as a corollary of the following (5, Theorem 3 ): "If $\Sigma$ is a closed subsemigroup of $P(S)$, then the minimal ideal $K(\operatorname{supp} \Sigma)$ of supp $\Sigma$ is the support of the minimal ideal $K(\Sigma)$ of $\Sigma$." Unfortunately the proof of this statement given in (5) contains a gap since the set

$$
\{\mu \in \Sigma: \operatorname{supp} \mu \cap K(\operatorname{supp} \Sigma) \neq \varnothing\}
$$

is not shown to be non-empty although essential use is made of the fact that it is an ideal of $\Sigma$. Here we give a direct proof of the " corollary " and deduce the " theorem ". (We note that Theorem 3 of (5) was used to derive several more results in (5), quoted in (8), and employed in the proof of Theorem 2 of (2).)

The author gratefully acknowledges the helpful advice of his supervisor, Dr G. Brown, and the financial support from the Association of Commonwealth Universities.

Proposition 1. Suppose $S$ is a compact semitopological semigroup. If $\Gamma$ is a simple subsemigroup of $P(S)$ and $\Gamma$ contains an idempotent $\eta$, then $S(\Gamma)$ is a topologically simple subsemigroup of $S$. 
Proof. Let $I$ be an ideal of $S(\Gamma)$ and let $\Omega=\{\mu \in \Gamma: \operatorname{supp} \mu \cap I \neq \varnothing\}$. It is easily seen that $\Omega$ is non-empty and an ideal of $\Gamma$ by virtue of $(4$, Lemma 4.1). This gives $\Omega=\Gamma$, whence supp $\eta \cap I \neq \varnothing$. Since supp $\eta$ is topologically simple, it follows that $\operatorname{supp} \eta \subset \bar{I}$. Hence for any measure $\mu \in \Gamma=\Gamma \eta \Gamma$, we have $\operatorname{supp} \mu=\overline{\operatorname{supp} \alpha \operatorname{supp} \eta \operatorname{supp} \beta} \subset \bar{I}$ for measures $\alpha, \beta \in \Gamma$. Consequently $S(\Gamma) \subset \tilde{I}$, i.e. $S(\Gamma)$ is topologically simple.

Example 2. In the preceding proposition, $S(\Gamma)$ need not be simple. For instance, take the semigroup $S$ given in (1, IV.7.1), i.e. $S=I \times I \times I$ with the usual topology, where $I=[0,1]$. Consider the separately continuous function $f: I \times I \rightarrow I$ defined by $f(x, z)=2 x z /\left(x^{2}+z^{2}\right)$ for $x, z$ not both zero, and $f(0,0)=0$. Then $S$ endowed with the multiplication

$$
(x, y, z)\left(x^{\prime}, y^{\prime}, z^{\prime}\right)=\left(x, f\left(x, z^{\prime}\right), z^{\prime}\right)
$$

is a compact semitopological semigroup. Its minimal ideal

$$
K(S)=\{(x, f(x, z), z): x, z \in I\}
$$

is not closed since $(0,1,0) \in \bar{K}(S) \backslash K(S)$. Now for any $a \in K(S)$ and $\mu \in P(S)$ we obtain the relation $\delta(a) \mu \delta(a)=\delta(a)(\delta(a)$ denotes the unit point mass at $a)$, since

$$
\delta(a) \mu \delta(a)(f)=\int f(a t a) d \mu(t)=\int f(a) d \mu(t)=f(a)=\delta(a)(f)
$$

for all $f \in C(S)$. It follows that the measure $\eta=\mu \delta(a) \mu$ is idempotent. If $\Gamma=\{\eta\}$ and $\mu$ is a measure with $S$ as its support (e.g. the restriction to $S$ of the Lebesgue measure on $\mathbf{R}^{3}$ ), then $S(\Gamma)=\operatorname{supp} \eta=\overline{S a S}=\bar{K}(S)$ which is not simple.

Example 3. With the hypotheses of Proposition 1, supp $\Gamma$ may not be topologically simple. For instance, take the one-point compactification $S=\mathbf{R} \cup\{\infty\}$ of $\mathbf{R}$ which is the additive group of real numbers with the operation extended by $x+\infty=\infty+x=\infty$ for $x \in S$. It follows that $S$ is a compact semitopological semigroup (1, IV.1.1.1.1(a)). Let $\Gamma=\{\delta(x): x \in \mathbf{R}\}$. Then $S(\Gamma)=\mathbf{R}$ and so supp $\Gamma=S$ which is clearly not topologically simple.

In the remainder of this paper, let $S$ be a compact jointly continuous semigroup. We have the next simple lemma which extends Lemma 1.3.11 of (6).

Lemma 4. Suppose $A$ is a subsemigroup of $S$. Then $A$ is topologically simple if and only if $\bar{A}$ is simple.

Proof. Let $\bar{A}$ be simple and take an ideal $I$ of $A$. It is obvious that $\bar{I}$ is an ideal of $\bar{A}$ and so $\bar{I}=\bar{A} \supset A$, i.e. $A$ is topologically simple. To show the converse, we model on the proof of (1, II.3.6). In fact for any $a \in A$ we see that $\bar{A} a \bar{A} \cap A \neq \varnothing$ is an ideal of $A$ and so $\bar{A} a \bar{A} \supset A$. Whence $\bar{A} a \bar{A}=\bar{A}$. Now we check that $\bar{A} x \bar{A}=\bar{A}$ for $x \in \bar{A}$. Clearly there exists a net $\left(x_{\alpha}\right)$ in $A$ such that $x_{\alpha} \rightarrow x$ and $\bar{A} x_{\alpha} \bar{A}=\bar{A}$. Let $b \in \bar{A}$ and we obtain two nets $\left(c_{\alpha}\right)$ and $\left(d_{\alpha}\right)$ in $\bar{A}$ 
such that $b=c_{\alpha} x_{\alpha} d_{\alpha}$. By passing to subnets if necessary, we have $c_{\alpha} \rightarrow c$ and $d_{a} \rightarrow d$, giving $b=c x d \in \bar{A} x \bar{A}$. It follows that $\bar{A} \subset \bar{A} x \bar{A}$. Thus $\bar{A}$ is simple.

Theorem 5. (1) If $\Gamma$ is a simple subsemigroup of $P(S)$, then supp $\Gamma$ is a compact simple subsemigroup of $S$.

(2) If $\Sigma$ is a closed subsemigroup of $P(S)$, then supp $K(\Sigma)=K(\operatorname{supp} \Sigma)$.

Proof. (1) Because supp $\bar{\Gamma}=\operatorname{supp} \Gamma$ (cf. (3), p. 55), we consider the compact semigroup $\bar{\Gamma}$ which contains an idempotent $\eta(6$, Theorem 1.1.10). Moreover, $\bar{\Gamma}$ is simple by Lemma 4 . Then it follows from Proposition 1 that $S(\bar{\Gamma})$ is topologically simple and so supp $\bar{\Gamma}$ is simple by Lemma 4 again. That supp $\Gamma$ is simple follows.

(2) Clearly supp $K(\Sigma)$ is an ideal of the semigroup supp $\Sigma$ and hence contains $K(\operatorname{supp} \Sigma)$. The result is now immediate.

Remark. In the theorem above, it is clear that $S(\Gamma)$ is a topologically simple semigroup, but we have been unable to determine whether this semigroup is always simple.

\section{REFERENCES}

(1) J. F. BERgLund and K. H. HofmAnN, Compact semitopological semigroups and weakly almost periodic functions (Lecture Notes in Mathematics 42, Springer-Verlag, Berlin-Heidelberg-New York, 1967).

(2) S. T. L. ChoY, On a limit theorem of measures, Math. Scand. 29 (1971), 256258.

(3) I. Glicksberg, Convolution semigroups of measures, Pacific J. Math. 9 (1959), 51-67.

(4) I. Glicksberg, Weak compactness and separate continuity, Pacific J. Math. 11 (1961), $205-214$.

(5) Y.-F. LIN, Not necessarily abelian convolution semigroups of probability measures, Math. Z. 91 (1966), 300-307.

(6) A. B. PaAlman-De MiRanda, Topological semigroups (Mathematisch Centrum, Amsterdam, 1964).

(7) J. S. PYM, Idempotent probability measures on compact semitopological semigroups, Proc. Amer. Math. Soc. 21 (1969), 499-501.

(8) J. H. Williamson, Harmonic analysis on semigroups, J. London Math. Soc. 42 (1967), 1-41.

UNIVERSITY OF LIVERPOOL

AND

Chung Chi College, Chinese University of Hong Kong

E.M.S. $-19 / 1-C$ 